California, USA. ${ }^{9}$ Institute of Biomaterials \& Bioengineering and ${ }^{10}$ Samuel Lunenfeld Research Institute, Department of Medical Genetics and Microbiology, University of

Toronto, Toronto, Canada. Correspondence should be addressed to W.C.S.

(skarnes@sanger.ac.uk).

1. Zambrowicz, B.P. et al. Nature 392, 608-611 (1998).

2. Zambrowicz, B.P. et al. Proc. Natl. Acad. Sci. USA 100, 14109-14114 (2003).
3. Zambrowicz, B.P. et al. Proc. Natl. Acad. Sci. USA 101, 4332-4333 (2004).

4. Valenzuela, D.M. et al. Nat. Biotechnol. 21, 652-659 (2003).

5. Hansen, J. et al. Proc. Natl. Acad. Sci. USA 100, 9918-9922 (2003)

\title{
Inauguration of the African Society of Human Genetics
}

\section{To the editor:}

In 2003, the African Society of Human Genetics (AfSHG) was established. The aim of AfSHG is to equip the African scientific community and policy-makers with the information and practical knowledge they need to contribute to the field of genetics research and to attract global attention to the efforts of African scientists. An important goal of this organization is to provide opportunities for networking and collaboration among professionals working on genetic and genomic issues relevant to Africa. By achieving these goals, AfSHG will help diminish the widening gap between Africa and the Western world in biomedical science.

The inaugural conference of AfSHG was held 8-9 December 2003 in Accra, Ghana. The theme for the meeting was

"Biomedical Research in Africa with Emphasis on Genetics". The meeting began with a review of socioeconomic and health conditions on the African continent. The importance of current realities for building a strong foundation for developing genetics and genomics in Africa was considered.

Genomic variation in the African diaspora was explored with attention to understanding the differential distribution of diseases, including malaria, tuberculosis, AIDS, diabetes, hypertension and cancer. Challenges surrounding the design and implementation of the only biobank in Africa, the Gambia National DNA Collections, were described. Current and potential applications of bioinformatics for the expansion of genetics and genomic research in Africa were reviewed. Geneenvironment interaction and its impact on the distribution of common complex diseases in Africa and the African diaspora were critically assessed. Ethical issues surrounding voluntary participation and informed consent in genetic research conducted in African settings and the current status of bioethics in medical education were discussed.
Future challenges for AfSHG. The fundamental goals of AfSHG are building capacity and networking. AfSHG plans to implement these objectives through several specific aims: (i) Information dissemination. The annual AfSHG conference and additional specialized workshops will provide a forum for disseminating and exchanging information through lectures and interactive training programs. (ii) Mentoring networks and educational resources. AfSHG will cultivate interest in investigative science and encourage African undergraduate and graduate students to pursue careers related to human genetics and biomedical sciences in general. (iii) Genetic advocacy. AfSHG will give voice to the cause of human genetics in Africa. AfSHG members will interface with policy-makers, the media and the public to help reduce fears and concerns surrounding genetics research. AfSHG will promote the design and implementation of genetics research that is ethically and culturally acceptable. (iv) Development of online courses. Members of AfSHG will develop online courses through affiliations with African, American and European universities and assist members with access to the internet. Additionally, programs will be initiated to diminish the widening North-South divide in biomedical science and technology. AfSHG will facilitate the participation of African scientists at annual international meetings of other professional organizations for genetics. (v) African Journal of Human Genetics. A scientific journal including research reports and critical reviews will be established to address specific interests of professionals working on genetics in the African context. This journal will supplement information available in textbooks and other resources, which are often scarce at many African institutions. (vi) Collaborative research. AfSHG will encourage and enhance collaborative research, which is currently responsible for $50 \%$ of the productivity of the African scientific community ${ }^{1}$. AfSHG will bring together foreign scientists working in Africa and African investigators to present information about populations of individuals with specific diseases, databases and technologies that would generate collaborative research. In addition to providing greater opportunities for research and exploration of genetics, applications for research grants and information about research sponsors will be made available. Courses in successful grant writing and training in clinical research methods, data management and manuscript development will be developed.

Membership in AfSHG is open to any individual who is interested in issues of human genetics in Africa, including but not limited to issues of education, clinical care, research, public health, ethics, law and policy. The field of genetics represents a continually expanding global enterprise. Successful international application of scientific and technological knowledge associated with genetics depends on cooperative arrangements between professionals living in diverse African countries and between Africans and their counterparts residing in other areas of the world. Collaborative partnerships in genomics and genetics will contribute greatly to promoting a sustained commitment to the development of the field of genetics in Africa. We envision AfSHG as the organization that will be a driving force in building a strong foundation for scientists and scholars working in genomics and genetics issues in Africa. Information on the AfSHG is available at http://www.afshg.org/.

\section{Charles N Rotimi}

National Human Genome Center, College of Medicine, Howard University, 2216 6th Street, NW, Suite 206, Washington, DC 20059, USA.

1. Jaffe, S. Back to Africa (Yes, it's possible to do science in Africa, but it's not that easy). The Scientist 16, 48-50 (2003). 